\title{
Braided Tensor Products and the Covariance of Quantum Noncommutative Free Fields
}

\author{
Jerzy Lukierski, Mariusz Woronowicz \\ Institute of Theoretical Physics \\ University of Wroclaw pl. Maxa Borna 9, 50 - 206 Wroclaw, Poland \\ e - mail : lukier@ift.uni.wroc.pl; woronow@ift.uni.wroc.pl
}

\begin{abstract}
We introduce the free quantum noncommutative fields as described by braided tensor product. The multiplication of such fields is decomposed into three operations, describing the multiplication in the algebra $\mathcal{M}$ of functions on noncommutative space-time, the product in the algebra $\mathcal{H}$ of deformed field oscillators, and the braiding by factor $\Psi_{\mathcal{M}, \mathcal{H}}$ between algebras $\mathcal{M}$ and $\mathcal{H}$. For noncommutativity of quantum space-time generated by the twist factor we shall employ the $\star$-product realizations of the algebra $\mathcal{M}$ in terms of functions on standard Minkowski space. The covariance of single noncommutative quantum fields under deformed Poincare symmetries is described by the algebraic covariance conditions which are equivalent to the deformation of generalized Heisenberg equations on Poincare group manifold. We shall calculate the braided field commutator covariant under deformed Poincare symmetries, which for free quantum noncommutative fields provides the field quantization condition and is given by standard Pauli-Jordan function. For ilustration of our new scheme we present explicit calculations for the well-known case in the literature of canonically deformed free quantum fields.
\end{abstract}

PACS numbers: $11.10 \mathrm{Nx}, 02.20 \mathrm{Uw}, 02.40 \mathrm{Gh}$

\section{Introduction}

It is at present a common view that the most important problem in the theory of fundamental interactions is to quantize in consistent way the dynamical theory of space-time, i.e. gravity, and incorporate it into the description of all other interactions. The consistent quantization of gravitation theory is searched in different ways: by employing new (nonlocal, discrete) descriptions of Einstein gravity (loop quantum gravity [1], spin foam models [2]) or by embedding standard gravity in larger dynamical framework ((super)string [3], brane world models [4]). In most of these approaches the classical commutative space-time geometry is modified because the quantization of gravitational dynamics leads to new algebraic structure of relativistic phase space with the noncommutative quantum space-time sector. In particular if we consider the quantum fields used e.g in the formulation of Standard Model in the presence of quantum gravity background, the appearance 
of quantum space-time implies that the standard framework for classical and quantum fields should be suitably deformed.

In recent fifteen years there were proposed various ways of introducing the deformation of quantum free fields which due to quantization procedure of gravity (see e.g. [5]) or quantized $D=10$ string effects in tensorial gauge fields background (see e.g. [6]) were defined on the noncommutative space-time [7]-[16]. It was proposed that the fields defined on relativistic space-time (Minkowski space) should be replaced by new fields which algebraically take into account the quantum gravity effects and are defined on quantum space-time. In these new field-theoretic models one replaces the commutative space-time coordinates $x_{\mu}$ by the noncommutative ones

$$
\left[x_{\mu}, x_{\nu}\right]=0 \quad \Longrightarrow \quad\left[\hat{x}_{\mu}, \hat{x}_{\nu}\right]=\frac{i}{\kappa^{2}} \Theta_{\mu \nu}(\kappa \hat{x})
$$

where $\left(\kappa\right.$ - masslike deformation parameter) $\Theta_{\mu \nu}(\kappa \hat{x})=\Theta_{\mu \nu}^{(0)}+\kappa \Theta_{\mu \nu}^{(1) \rho} \widehat{x}_{\rho}+\cdots$. The choice $\Theta_{\mu \nu}(\kappa \widehat{x})=\Theta_{\mu \nu}^{(0)}$ corresponds to the so-called canonical deformation [5], [6] and $\Theta_{\mu \nu}(\kappa \widehat{x})=$ $\Theta_{\mu \nu}^{(1) \rho} \widehat{x}_{\rho}$ provides the Lie-algebraic noncommutativity of space-time with the most known special case describing the $\kappa$-deformation of Minkowski space [17]-[19]. It has been also shown in the examples of deformed quantum free fields that the noncommutativity of space-time algebra is linked in various ways with the deformation of field oscillators algebra (see e.g. [20]-[28]).

One introduces the symmetries in theories with noncommutativity (1) in two distinct ways:

1. One keeps the classical Poincare symmetries and the noncommutativity (1) is treated as introducing the terms which break the classical relativistic symmetries [5],[29][31]. In such a way the choice of function $\Theta_{\mu \nu}(\kappa \hat{x})$ can be arbitrary (modulo Jacobi identities) and the set of constant tensors $\Theta_{\mu \nu}{ }^{\rho_{1} \ldots \rho_{n}}(\rho=0,1,2 \ldots)$ describe the classical Poincare symmetry breaking parameters.

2. One can introduce the modified quantum Poincare-Hopf symmetries in a way which leads to the covariance of the relations (11) (see e.g. [12]-[16]). In such approach quantum covariance means that the relation (11) has the same algebraic form in all deformed Poincare frames and $\Theta_{\mu \nu}^{(n) \rho_{1} \ldots \rho_{n}}(n=0,1,2 \ldots)$ can be treated as set of constant parameters, which also enter into the deformed Poincare-Hopf algebra. It appears that such method is more restrictive, it selects only the particular classes of functions $\Theta_{\mu \nu}(\kappa \hat{x})$ occuring in (1).

In this paper we shall follow the second approach.

The basic aim of this paper is to study new framework for description of covariant twist-deformed free quantum fields, with the covariant deformation of field oscillators algebra. It is known already from earlier studies of Faddeev-Zamolodchikov and $q$-deformed Heisenberg algebras (see e.g. [32]-[36]) that the deformed commutators of field oscillators which are covariant under the quasitriangular quantum symmetry takes a braided 
form, with the covariant braid factor described by corresponding universal $\mathcal{R}$-matrix (see e.g. [37], Sect. 7.2). Such $\mathcal{R}$-matrix-dependent braid factor will also characterize the c-number commutator of deformed free quantum fields as well as the braided products of these fields.

In Sect. 2 we describe the properties of general twisting procedure of Hopf algebras and specify the description for the canonical twist. In Sect. 3 we shall consider the deformed noncommutative free quantum fields $\phi(\widehat{x})$ as described by binary braided tensor products $\mathcal{M} \otimes \mathcal{H}$ where $\mathcal{M}$ is the algebra of functions on noncommutative space-time and $\mathcal{H}$ denotes the algebra of deformed field oscillators (see also [38]-[40], where however the unbraided tensor product is used). We point out that the nontrivial braiding between quantum symmetry modules $\mathcal{M}$ and $\mathcal{H}$ will be only revealed in the multiplication procedure of quantum fields. In Sect. 4 we describe two covariant actions of the deformed Poincare generators on a deformed quantum field. In the definition of single noncommutative quantum field after introduction in $\mathcal{M}$ of the Weyl map the tensor structure $\mathcal{M} \otimes \mathcal{H}$ collapses into the field oscillators algebra module $\mathcal{H}$, what is also required by the correct no-deformation limit of the deformed quantum fields. Algebraically the covariance condition will be expressed on deformed fields as generalized Heisenberg field equations. Further in Sect. 5 we shall consider the products of deformed free fields with the braided multiplication $m_{\bullet}$. Such new multiplication rule introduces the braiding factor $\Psi_{\mathcal{M}, \mathcal{H}}$ between the algebra of space-time functions and deformed field oscillators (see also Fiore and Wess [15], [28, [36] $]$ ). If the deformation of Poincare symmetries is described by universal $\mathcal{R}$-matrix (we denote $\mathcal{R}=\mathcal{R}_{(1)} \otimes \mathcal{R}_{(2)}$ and $\mathcal{R}_{21}=\mathcal{R}_{(2)} \otimes \mathcal{R}_{(1)}$ ) the braid factor $\Psi_{\mathcal{M}, \mathcal{H}}$ will be defined by $\mathcal{R}_{21}$ and the quantization of free quantum field $\phi \in \mathcal{M} \otimes \mathcal{H}$ will be described by the covariant braided commutator 2

$$
[\phi(\widehat{x}), \phi(\widehat{y})]_{\bullet}^{B R} \equiv \phi(\widehat{x}) \bullet \phi(\widehat{y})-\mathcal{R}_{21} \bullet(\phi(\widehat{y}) \bullet \phi(\widehat{x}))
$$

where we shall argue in Sect. 4 that the action $g \boldsymbol{\phi}$ of deformed Poincare algebra generators occurring in $\mathcal{R}_{21}$ is fully described in covariant theory by the operation $>$ of quantum adjoint in the algebra $\mathcal{H}$ of deformed field oscillators. Our basic point is here (see also Aschieri at all in [42]) that the deformed Poincare covariance of the free quantum field commutator requires the braided form (2).

In the paper we restrict our explicit considerations to the triangular deformations described by Drinfeld twist [43]. Our main aim is to show that using suitable twist-covariant braided algebra of deformed field oscillators we get the c-number braided field commutator (2) describing covariant algebra of quantum noncommutative free fields. Because twist deformation of Poincare symmetries does not modify the mass-shell condition, such braided commutator of twist-deformed quantum free fields is given by the standard relativistic Pauli-Jordan commutator function.

\footnotetext{
${ }^{1}$ Similar braiding factor has been considered also in [41] but for different aims.

${ }^{2}$ We introduced the short-hand notation. The operator $\mathcal{R}_{21}$ acts in the definition (2) of braided commutator as follows$$
\mathcal{R}_{21} \triangleright(\phi(\widehat{y}) \bullet \phi(\widehat{x})) \equiv m_{\bullet} \circ \mathcal{R}_{21} \triangleright(\phi(\widehat{y}) \otimes \phi(\widehat{x}))=\left(\mathcal{R}_{(2)} \triangleright \phi(\widehat{y})\right) \bullet\left(\mathcal{R}_{(1)} \triangleright \phi(\widehat{x})\right) .
$$ 
In order to illustrate our approach we shall consider in explicit way the simple example of canonically deformed QFT $\left(\Theta_{\mu \nu}(\kappa \widehat{x})=\Theta_{\mu \nu}^{(0)}\right.$ in (1)), with Weyl map described by Moyal-Weyl «-product. It can be also shown (see e.g. [45]), that important class of noncanonically deformed relations (11) with rhs containing the linear and quadratic terms can be made covariant under twisted Poincare symmetries. On such class of noncommutative space-times one can define as well the corresponding deformed free quantum fields with covariant braided field commutator (2). We conclude that our scheme provides the description of deformed free QFT which is covariant under twisted Hopf-Poincare algebra but in principle can be extended to the deformations described by any quasitriangular universal $\mathcal{R}$-matrix.

\section{The quantum Poincare-Hopf algebras and the de- formation by canonical twist}

The classical relativistic symmetries are generated by Poincare Lie algebra $\mathcal{P}$, which can be endowed with trivial (primitive) coalgebraic structure by the coproducts $\left(g=\left(M_{\mu \nu}, P_{\mu}\right)\right)$

$$
\Delta_{0}(g)=g \otimes 1+1 \otimes g,
$$

describing the homomorphic map $\Delta_{0}: \mathcal{U}_{0}(\mathcal{P}) \rightarrow \mathcal{U}_{0}(\mathcal{P}) \otimes \mathcal{U}_{0}(\mathcal{P})$ of enveloping classical Poincare algebra $\mathcal{U}_{0}(\mathcal{P})$. For the description of quantum relativistic symmetries one uses the quantum Poincare-Hopf algebras $\mathbb{H}(\mathcal{U}(\mathcal{P}), m, \eta, \Delta, \epsilon, S)$, where $\mathcal{U}(\mathcal{P})$ describes the enveloping deformed Poincare algebra with multiplication map $m: \mathcal{U}(\mathcal{P}) \otimes \mathcal{U}(\mathcal{P}) \rightarrow \mathcal{U}(\mathcal{P})$; $\Delta: \mathcal{U}(\mathcal{P}) \rightarrow \mathcal{U}(\mathcal{P}) \otimes \mathcal{U}(\mathcal{P})$ denotes the nonprimitive (noncommutative) coproduct map and $S: \mathcal{U}(\mathcal{P}) \rightarrow \mathcal{U}(\mathcal{P})$ defines the antipode or coinverse, which for classical Lie algebras is given by $S_{0}(g)=-g$. For complete definition of a Hopf algebra (see e.g. [50]) one introduces also the unit $\eta(m(\eta \otimes a)=a)$ and counit $\epsilon$, playing the role of unit for coalgebra maps.

A simple way of passing from classical to quantum Poincer-Hopf algebra is achieves by introducing the twist $\mathcal{F}$. During twisting procedure of undeformed Poincare Hopf algebra the algebraic sector remains unchanged, therefore the classical Poincare algebra remains valid and the formulae for Casimirs are not changed. The twist factor $\mathcal{F} \in \mathcal{U}(\mathcal{P}) \otimes \mathcal{U}(\mathcal{P})$ deforms only the co-structure of Hopf algebra by modification of coproduct and antipode by the following formulae

$$
\begin{aligned}
& \Delta^{\mathcal{F}}(g)=\mathcal{F} \Delta_{0}(g) \mathcal{F}^{-1}, \\
& S^{\mathcal{F}}=v S_{0}(g) v^{-1}=-v g v^{-1}, \quad v=f_{(1)} S\left(f_{(2)}\right),
\end{aligned}
$$

where we denote $\left.\mathcal{F}=f_{(1)} \otimes f_{(2)}\right]^{3}$. When the coproduct is changed the action of algebra $\mathcal{U}(\mathcal{P})$ on tensor product representation is modified [50] because the coproduct (4) enters into the covariant action as follows $\left(\Delta^{\mathcal{F}}(g)=g_{(1)} \otimes g_{(2)}\right)$

$$
g \triangleright(v w)=m\left[\Delta^{\mathcal{F}}(g) \triangleright(v \otimes w)\right]=\sum\left(g_{(1)} \triangleright v\right)\left(g_{(2)} \triangleright w\right), \quad g \triangleright 1=\epsilon(g) \triangleright 1 .
$$

\footnotetext{
${ }^{3}$ We use Sweedler notation with suppresing the summ index.
} 
If twist $\mathcal{F}$ does not generate nontrivial coassociator it should satisfy the 2-cocycle condition

$$
\mathcal{F}_{12}(\Delta \otimes i d) \mathcal{F}=\mathcal{F}_{23}(i d \otimes \Delta) \mathcal{F}
$$

In such a case the leading term describing twist factor $\mathcal{F}$ is described by the classical $r$-matrix (by $\xi$ we denote the deformation parameter)

$$
\mathcal{F}=1 \otimes 1+\xi r+\mathcal{O}\left(\xi^{2}\right)
$$

where $r \in g \wedge g$ satisfies classical Yang-Baxter equation (CYBE), one gets for any generator $g$ that

$$
\Delta(g)=\Delta^{(0)}(g)+\xi\left[r, \Delta^{(0)}(g)\right]+\mathcal{O}\left(\xi^{2}\right) .
$$

From Lie algebra relations for $g$ follows that the first order correction in (9) belongs to the tensor product $g \otimes g$.

The algebra of the canonically deformed space-time

$$
\left[\hat{x}_{\mu}, \hat{x}_{\nu}\right]=i \theta_{\mu \nu}
$$

can be described as covariant under deformed (twisted) Poincare symmetries if one introduces the canonical twist factor [8, [12, [13]

$$
\mathcal{F}=e^{\frac{i}{2} \theta^{\mu \nu} P_{\mu} \otimes P_{\nu}} .
$$

For canonical deformation, from twist factor (11), we obtain only the modification of coproduct for Lorentz generators $M_{\mu \nu}$

$$
\begin{aligned}
\Delta^{\mathcal{F}}\left(P_{\mu}\right) & =\Delta_{0}\left(P_{\mu}\right), \\
\Delta^{\mathcal{F}}\left(M_{\mu \nu}\right) & =\Delta_{0}\left(M_{\mu \nu}\right)-\theta^{\rho \sigma}\left[\left(\eta_{\rho \mu} P_{\nu}-\eta_{\rho \nu} P_{\mu}\right) \otimes P_{\sigma}\right. \\
& \left.\quad+P_{\rho} \otimes\left(\eta_{\sigma \mu} P_{\nu}-\eta_{\sigma \nu} P_{\mu}\right)\right] .
\end{aligned}
$$

The coinverse as well as counit remain undeformed

$$
S(g)=-g, \quad \epsilon(g)=0 .
$$

For twisted canonical space-time generated by twist (11), the multiplication $\star_{\mathcal{M}}$ (21) in the algebra $\mathcal{M}$ is defined by the twist factor $\mathcal{F}$ as follows

$$
e^{i p x} \star_{\mathcal{M}} e^{i q x}=m \circ \mathcal{F}^{-1} \triangleright\left[e^{i p x} \otimes e^{i q x}\right]=m \circ\left(\bar{f}_{(1)} \triangleright e^{i p x} \otimes \bar{f}_{(2)} \triangleright e^{i q y}\right)=\mathcal{F}^{-1}(p, q) e^{i p x} e^{i q x},
$$

where in general case we denote $\mathcal{F}^{-1}=\bar{f}_{(1)} \otimes \bar{f}_{(2)}$ and in canonical case $\mathcal{F}^{-1}(p, q)=$ $e^{-\frac{i}{2} p \theta q}, p \theta q \equiv p^{\mu} \theta_{\mu \nu} q^{\nu}$.

The algebra (10) can be extended consistently to the collection of noncommutative space-time coordinates $\left(\widehat{x}_{\mu}^{(1)}, \widehat{x}_{\mu}^{(2)} \ldots \widehat{x}_{\mu}^{(n)}\right)$ in the following way [15], [40] $(i, j=1,2, \ldots, n)$

$$
\left[\widehat{x}_{\mu}^{(i)}, \widehat{x}_{\mu}^{(j)}\right]=i \theta_{\mu \nu} .
$$


If we introduce the corresponding Weyl map for bilocal product $e^{i p \widehat{x}} e^{i q \widehat{y}}\left(\widehat{x}_{\mu} \equiv \widehat{x}_{\mu}^{(1)}, \widehat{y}_{\mu} \equiv\right.$ $\left.\widehat{x}_{\mu}^{(2)}\right)$ it can be shown that the formula (15) can be extended as follows

$$
e^{i p x} \star_{\mathcal{M}} e^{i q y}=m \circ \mathcal{F}^{-1} \triangleright\left[e^{i p x} \otimes e^{i q y}\right]=\mathcal{F}^{-1}(p, q) e^{i p x} e^{i q y}
$$

The twisted canonical Poincare algebra is the triangular Hopf algebra, with universal $\mathrm{R}$ matrix satisfying the relation

$$
\Delta_{21}=\mathcal{R} \Delta \mathcal{R}^{-1}
$$

and given by the formula

$$
\mathcal{R}=\mathcal{F}_{21} \mathcal{F}^{-1}=\mathcal{F}^{-2}, \quad \mathcal{R}_{21}=\mathcal{R}^{-1}=\mathcal{F}^{2}
$$

where in (19) we used the relation $\mathcal{F}_{21}=\mathcal{F}^{-1}$. The formulae (19) describe the universal $\mathcal{R}$-matrix for triangular Hopf algebras.

\section{Deformed quantum fields and braided tensor prod- uct}

If we construct deformed QFT we deal with the following three algebras: the algebra $\mathcal{M}$ of functions $f \in \mathcal{M}$ on noncommutative space time, the algebra $\mathcal{H}$ of deformed field oscillators $h \in \mathcal{H}$ and the algebra $\Phi$ of deformed fields $\phi \in \Phi$. The deformed relativistic free quantum fields $\phi$ can be described as the infinite sum (in fact continuous integral) of the braided tensor products of the noncommutative plane waves $e^{i p \widehat{x}}$ from $\mathcal{M}$ and the elements $A(p)$ from the algebra of field oscillators $\mathcal{H}$

$$
\phi \in \mathcal{M} \underline{\otimes} \mathcal{H}
$$

where underlining of the tensor product described its braided nature.

The algebra $\mathcal{M}\left(e^{i p \widehat{x}}, \cdot\right)$ of basic functions on noncommutative space-time $\widehat{x}=\left(\widehat{x}_{i}, \widehat{x}_{0}\right)$ we shall further represent izomorphically by the star product $\star_{\mathcal{M}}$ of commutative functions $e^{i p x}$ providing by means of the Weyl map $\mathcal{M}\left(e^{i p x}, \star_{\mathcal{M}}\right)$

$$
\begin{aligned}
e^{i p \widehat{x}} e^{i q \widehat{x}}= & m\left(e^{i p \widehat{x}} \otimes e^{i q \widehat{x}}\right) \\
& \stackrel{\text { Weyl map }}{\longrightarrow} m_{\mathcal{M}}\left(e^{i p x} \otimes e^{i q x}\right)=e^{i p x} \star_{\mathcal{M}} e^{i q x},
\end{aligned}
$$

where $x=\left(x_{i}, x_{0}\right)$ describes standard Minkowski space-time coordinates. We add that for the consideration of bilocal products $\phi(\widehat{x}) \phi(\widehat{y})$ we should introduce as well the Weyl map $e^{i p \widehat{x}} e^{i q \widehat{y}} \longrightarrow e^{i p x} \star_{\mathcal{M}} e^{i q y}$ generalizing the formula (21) (see also (15) and (17)). Effectively in formula (20) we shall describe $\mathcal{M}$ by the commutative functions on classical Minkowski space with the modified star-multiplication law $m \rightarrow m_{\mathcal{M}}$.

The Poincare algebra as well as its Casimirs are not modified by the twist deformation, i.e. if we consider only twisted Poincare symmetries they do not change the mass-shell which is provided by the mass Casimir, or equivalently the relativistic energy-momentum 
dispersion relation $\omega(\vec{p})=\sqrt{\vec{p}^{2}+m^{2}}$ remain valid after twisting. In accordance with (20), in twisted field theory one can use the standard Fourier decomposition of free scalar quantum fields with noncommutative Fourier exponentials

$$
\begin{aligned}
\phi(\widehat{x}) & =\frac{1}{(2 \pi)^{4}} \int d^{4} p \delta\left(p^{2}-m^{2}\right) \mathrm{e}^{i p \widehat{x}} \otimes A(p) \\
& =\frac{1}{(2 \pi)^{3}} \int \frac{d^{3} \vec{p}}{2 \omega(\vec{p})}\left(\mathrm{e}^{i p \widehat{x}} \otimes a^{\dagger}(\vec{p})+\mathrm{e}^{-i p \widehat{x}} \otimes a(\vec{p})\right)_{p_{0}=\omega(\vec{p})},
\end{aligned}
$$

where $a(\vec{p})=A(\vec{p}, \omega(\vec{p})),\left(a^{\dagger}(\vec{p})=A(-\vec{p},-\omega(\vec{p}))\right)$ are the deformed annihilation (creation) field oscillator.

The algebra of deformed field oscillators $\mathcal{H}(A(p) ; \cdot)$ with standard multiplication in $\mathcal{H}$ $(m(A(p) \otimes A(q))=A(p) A(q))$ in noncommutative case will be described in accordance with the quantum-deformed covariance condition by the braided commutation relations of oscillators algebra with suitable multiplication in $\mathcal{H}$.

If we use the Weyl map (21) the elements of algebra $\mathcal{M}$ are replaced by classical functions (e.g. $e^{i p \widehat{x}} \rightarrow e^{i p x}$ ). Further using the isomorphism $\mathbb{C} \otimes \mathcal{H} \simeq \mathcal{H}$ one can introduce field $\phi(\widehat{x}) \stackrel{W}{\simeq} \varphi(x)$ (W denotes Weyl map) having well-known standard form

$$
\begin{aligned}
\varphi(x) & =\frac{1}{(2 \pi)^{4}} \int d^{4} p \delta\left(p^{2}-m^{2}\right) \mathrm{e}^{i p x} A(p) \\
& =\frac{1}{(2 \pi)^{3}} \int \frac{d^{3} \vec{p}}{2 \omega(\vec{p})}\left(\mathrm{e}^{i p x} a^{\dagger}(\vec{p})+\mathrm{e}^{-i p x} a(\vec{p})\right)_{p_{0}=\omega(\vec{p})},
\end{aligned}
$$

where $\varphi(x) \in \mathcal{H}$, and we point out that oscillators $A(p)$ will satisfy the deformed binary relations.

The tensor structure of the deformed quantum field (see (20) and (22)) should be consistent with the actions of the space-time symmetry generator $g$. On the deformed symmetry algebra modules $\mathcal{M}$ and $\mathcal{H}$ the actions of $g$ is defined by the coproduct $\Delta(g)=$ $g_{(1)} \otimes g_{(2)}$ as follows

$$
\begin{aligned}
& g \triangleright a=a d_{g} a=\sum g_{(1)} a S\left(g_{(2)}\right) . \quad a \in \mathcal{H}, \\
& g \triangleright f=a d_{g} f=\widehat{D}(g) f . \quad f \in \mathcal{M},
\end{aligned}
$$

where $\widehat{D}(g)$ describes the differential realization of $g$ on noncommutative functions $f \in \mathcal{M}$.

If we wish to define the actions $\triangleright$ and $\triangleright$ on the tensor product $\mathcal{M} \otimes \mathcal{H}$ we postulate in consistency with the symmetry properties of no-deformation classical limit the trivial actions on "wrong" part of the tensor product (20)

$$
\begin{aligned}
g \triangleright f & =\epsilon(g) f, \\
g \triangleright a & =\epsilon(g) a,
\end{aligned}
$$


where $\epsilon(1)=1$ and $\epsilon(g)=0$ for any Poincare generators. We see that modules $\mathcal{M}$ under action $\$ and the module $\mathcal{H}$ under the action $\triangleright$ behaves as number (scalar spectator). In particular e.g. if we choose the product of basic modules $e^{i p \widehat{x}} \underline{\otimes} a(\vec{p})$ one gets for example

$$
\begin{aligned}
g \triangleright\left(e^{i p \widehat{x}} \underline{\otimes} a(\vec{p})\right) & =(a d \otimes a d) \Delta^{\mathcal{F}}(g)\left(e^{i p \widehat{x}} \underline{\otimes} a(\vec{p})\right) \\
& =a d_{g_{(1)}} e^{i p \widehat{x}} \underline{\otimes} a d_{g_{(2)}} a(\vec{p})=\left(g_{(1)} \triangleright e^{i p \widehat{x}}\right) \underline{\otimes}\left(g_{(2)} \triangleright a(\vec{p})\right) .
\end{aligned}
$$

Because the general coproduct can be written as $\left(\Delta^{(0)}(g)=g \otimes 1+1 \otimes g\right.$; see also (91))

$$
\Delta^{\mathcal{F}}(g)=\Delta^{(0)}(g)+\text { terms not containing components } 1 \otimes g \text { and } g \otimes 1,
$$

the only term contributing to $g_{(1)}>e^{i p \widehat{x}}$ comes from $1 \otimes g$, and one gets

$$
g \triangleright\left(e^{i p \widehat{x}} \underline{\otimes} a(\vec{p})\right)=\left(1 \triangleright e^{i p \widehat{x}}\right) \underline{\otimes}(g \triangleright a(\vec{p}))=e^{i p \widehat{x}} \underline{\otimes}(g \triangleright a(\vec{p})) .
$$

The relation (30) has the correct classical limit when the factor $e^{i p \widehat{x}}$ becomes a classical function $e^{i p x}$. Similarly

$$
g \triangleright\left(e^{i p \widehat{x}} \underline{\otimes} a(\vec{p})\right)=\left(g \triangleright e^{i p \widehat{x}}\right) \underline{\otimes} a(\vec{p}) .
$$

In the consideration of deformed symmetry properties we shall need only the actions (30), (31) of the generators $g$.

In this paper we consider the extension of the usual framework by introducing the algebra of fields $\phi$ with braided multiplication rule. We should multiply the noncommutative fields (20) in a way taking into account the braid $\Psi_{\mathcal{M}, \mathcal{H}}$ introduced as follows

$$
\begin{aligned}
\phi(\widehat{x}) \bullet \phi(\widehat{y}) & =m_{\mathcal{M} \otimes \mathcal{H}} \phi(\widehat{x}) \otimes \phi(\widehat{y}) \\
& =(m \otimes m) \circ\left(i d \otimes \Psi_{\mathcal{M}, \mathcal{H}} \otimes i d\right) \phi(\widehat{x}) \otimes \phi(\widehat{y}) .
\end{aligned}
$$

Braid factor $\Psi_{\mathcal{M}, \mathcal{H}}$ describes effectively the noncommutativity of factors $A(p)$ and $e^{i q \widehat{y}}$ in the product of field operators in accordance with general formula for covariant braiding acting on two modules of quasitriangular Hopf algebra. It is defined by universal matrix $\mathcal{R}=\mathcal{R}_{1} \otimes \mathcal{R}_{2}(f \in \mathcal{M}, h \in \mathcal{H})$; see e.g. [37]

$$
\Psi_{\mathcal{M}, \mathcal{H}}(h \underline{\otimes} f)=\left(\mathcal{R}_{2} \triangleright f\right) \underline{\otimes}\left(\mathcal{R}_{1} \triangleright h\right) .
$$

We see that if wespecify in $\mathcal{M} \underline{\otimes \mathcal{H}}$ the braid factor $\mathcal{R}_{21}$, the generators $g$ (we recall that $\mathcal{R}$ is defined in terms of Poincare algebra generators $g$ ) act nontrivially on both legs of the tensor product. In fact before applying Weyl map to the product (32) we should firstly use braid factor $\Psi_{\mathcal{M}, \mathcal{H}}$ in order to introduce ordered elements of $\mathcal{M}$ and $\mathcal{H}$, with field oscillators on the right and noncommutative Fourier exponentials on the left.

The covariant braid factor (33) will be necessary in order to introduce the multiplication in the covariant algebra of deformed quantum fields $\Phi\left(\phi, m_{\mathcal{M} \underline{\otimes \mathcal{H}}}\right)$. The commutative (undeformed) limit of the relation (32) is obtained by putting $\Psi_{\mathcal{M}, \mathcal{H}}=\tau$, where $\tau$ is the flip operator describing no deformation limit of $\mathcal{R}_{21}$. In standard Poincare symmetry case one assumes that

$$
\left[e^{i p x}, A(q)\right]=(1-\tau) e^{i p x} A(q)=0 .
$$


i.e. the standard free quantum fields can be described by unbraided tensor products $\left(m_{\mathcal{M} \otimes \mathcal{H}} \longrightarrow m_{\mathcal{M} \otimes \mathcal{H}}\right)$ which provides the ordering

$$
m_{\mathcal{M} \underline{\otimes} \mathcal{H}}\left[\left(e^{i p x} \underline{\otimes} A(p)\right) \otimes\left(e^{i q y} \underline{\otimes} A(q)\right)\right]=e^{i p x} e^{i q y} \underline{\otimes} A(p) A(q) \simeq e^{i(p x+q y)} A(p) A(q) .
$$

In this paper we describe the algebra of deformed quantum free fields with noncommutative space-time arguments endowed with nontrivial braided multiplication (32) consistent with twisted Poincare covariance. The deformed covariant field quantization will be described by the $c$-number braided field commutator (see Sect. 5). We shall perform the explicit calculations for the case of quantum deformation described by the canonical twist (11). Firstly in following Section we shall describe the covariance conditions satisfied by twist-deformed quantum fields.

\section{The twisted covariance of single quantum field}

Let us consider firstly the Poincare covariance of the standard (undeformed) free quantum field $\varphi(x)$, given by (23) with algebra $\mathcal{H}$ describing standard field oscillators algebra. The classical Poincare covariance is given by the known formula

$$
U(\Lambda, a) \varphi(x) U^{-1}(\Lambda, a)=\varphi(\Lambda x+a),
$$

where $U(\Lambda, a)=\exp \left(i a^{\mu} P_{\mu}+i \omega^{\mu \nu} M_{\mu \nu}\right)$ describes the unitary representation of Poincare group in Hilbert-Fock space, generated from the Poincare-invariant vacuum by the products of field oscillators. For infinitesimal $a_{\mu}$ and $\omega_{\mu \nu}\left(\Lambda_{\nu}^{\mu}=\delta_{\nu}^{\mu}+\omega_{\nu}^{\mu}\right)$ from relation (36) follows that there are two ways of expressing the action of Poincare generators on quantum free field which give the same result

$$
\begin{aligned}
P_{\mu} \triangleright \varphi & :=a d_{P_{\mu}}^{(0)} \varphi=\left[P_{\mu}, \varphi\right]=-D^{(0)}\left(P_{\mu}\right) \varphi=:-P_{\mu} \triangleright \varphi, \\
M_{\mu \nu} \triangleright \varphi & :=a d_{M_{\mu \nu}}^{(0)} \varphi=\left[M_{\mu \nu}, \varphi\right]=-D^{(0)}\left(M_{\mu \nu}\right) \varphi=:-M_{\mu \nu} \triangleright \varphi .
\end{aligned}
$$

We see that the relations (37)-(38) can be written as

$$
g^{(0)} \triangleright \varphi=-g^{(0)} \triangleright \varphi
$$

where $g^{(0)}=$ denotes the undeformed Poincare algebra generators provide the generalized Heisenberg equation on the classical Poincare group manifold.

In undeformed quantum free field case the generators $P_{\mu}, M_{\mu \nu}$ are the bilinear functionals of the oscillators $a(p), a^{\dagger}(p)$ and on rhs of (37)-(38) we have the known relativistic differential realizations $D^{(0)}\left(g_{A}\right)\left(g_{A}=\left(P_{\mu}, M_{\mu \nu}\right)\right)$ of standard Poincare-Hopf algebra on the scalar fields with Minkowski space-time arguments

$$
D^{(0)}\left(P_{\mu}\right)=-i \partial_{\mu}, \quad D^{(0)}\left(M_{\mu \nu}\right)=-i\left(x_{\mu} \partial_{\nu}-x_{\nu} \partial_{\mu}\right) .
$$

The generalized Heisenberg eq. (37)-(38) describe in infinitesimal form the standard global Poincare covariance of quantum free fields, given by relation (36). 
In noncommutative framework the description should be modified in accordance with formula (20) and corresponding Hopf-algebraic language. The deformed counterpart of the equations (37) and (38) should be derived with the use of the formulae (28)-(30). If we observe that the twisted coproduct is given by the formula (9), due to the vanishing values of counit $\epsilon(g)$ only $\Delta^{(0)}(g)$ in (9) will contribute to the adjoint action $g \triangleright \phi$ in accordance with (29). We obtain therefore in deformed case the following deformed generalized Heisenberg equations

$$
g \triangleright \phi=S(g) \triangleright \phi, \quad g=\left(P_{\mu}, M_{\mu \nu}\right)
$$

or more explicitly

$$
\begin{aligned}
P_{\mu} \triangleright \phi & =\left(a d_{P_{\mu(1)}} \otimes a d_{P_{\mu(2)}}\right) \phi=\left(1 \otimes a d_{P_{\mu}}\right) \phi=\left(D\left[S\left(P_{\mu}\right)\right] \otimes 1\right) \phi=S\left(P_{\mu}\right) \triangleright \phi, \quad(42) \\
M_{\mu \nu} \triangleright \phi & \left.=\left(a d_{M_{\mu \nu(1)}} \otimes a d_{M_{\mu \nu(2)}}\right) \phi=\left(1 \otimes a d_{M_{\mu \nu}}\right) \phi=\left(D\left[S\left(M_{\mu \nu}\right)\right] \otimes 1\right) \phi=S\left(M_{\mu \nu}\right) \text { 中4ß) }\right)
\end{aligned}
$$

where the adjoint action of deformed symmetry generators $g$ in $\mathcal{H}$ are given by (24) and $\widehat{D}(g)$ is the suitably deformed differential realization on $\mathcal{M}$ of standard Poincare algebra, consistent with deformed coalgebra structure. In particular after Weyl map $f(\widehat{x}) \stackrel{W}{\rightarrow} f(x)$, $g(\widehat{x}) \stackrel{W}{\rightarrow} g(x), \widehat{D}(g) \stackrel{W}{\rightarrow} D(g)$ we should have the following form of modified Leibnitz rule

$$
D(g)\left(f(x) \star_{\mathcal{M}} g(x)\right)=m_{\mathcal{M}} \circ\left[(D \otimes D) \Delta^{\mathcal{F}}(g) \circ f(x) \otimes g(x)\right] .
$$

In the case of canonical deformation, as follows from (12), we obtain undeformed covariance relation (37) for the momentum generators, because from (24) follows that

$$
\left(1 \otimes a d_{P_{\mu}}\right) \phi=\left(1 \otimes a d_{P_{\mu}}^{(0)}\right) \phi,
$$

and $D\left(P_{\mu}\right)=D^{(0)}\left(P_{\mu}\right)$. For the Lorentz algebra generators $M_{\mu \nu}$ it follows from (13), (14) and (24) that

$$
\left(1 \otimes a d_{M_{\mu \nu}}\right) \phi=\left[1 \otimes M_{\mu \nu}, \phi\right]+\theta_{[\nu}{ }^{\alpha}\left(1 \otimes P_{\alpha}\right) \phi\left(1 \otimes P_{\mu]}\right)+\theta_{[\mu}{ }^{\alpha}\left(1 \otimes P_{\nu]}\right) \phi\left(1 \otimes P_{\alpha}\right) .
$$

In order to obtain in relation (43) the suitably deformed differential realization of Lorentz algebra generators $D\left(M_{\mu \nu}\right)$ one can use or differential realization on noncommutative Minkowski space or after performing the Weyl map we consider the deformation $D\left(M_{\mu \nu}\right)$ of $D^{(0)}\left(M_{\mu \nu}\right)$ (see (40) ) on standard Minkowski space extended to relativistic phase space. We shall use below the second possibility. Let us observe that the noncommutative space-time coordinates (10) can be described by the relativistic nondeformed quantum phase space variables $\left(x_{\mu}, p_{\mu}\right)$ as follows

$$
\widehat{x}_{\mu}=x_{\mu}+\theta_{\mu \nu} p^{\nu}
$$

where $\left[x_{\mu}, p_{\nu}\right]=i \eta_{\mu \nu}\left(\eta_{\mu \nu}=\operatorname{diag}(-1,1,1,1)\right),\left[x_{\mu}, x_{\nu}\right]=\left[p_{\mu}, p_{\nu}\right]=0$. Using relation (47) and the standard Lorentz transformations of the variables $x_{\mu}, p_{\nu}$, the modified Lorentz transformation of canonically deformed space-time variables $\widehat{x}_{\mu}$ can be expressed as

$$
\widehat{x}_{\mu}^{\prime}=\Lambda_{\mu}^{\nu} x_{\nu}+\theta_{\mu \nu} \Lambda_{\rho}^{\nu} p^{\rho}=\Lambda_{\mu}^{\nu} \widehat{x}_{\nu}+\widehat{\xi}^{\nu}
$$


where $\widehat{\xi}^{\nu}=\left(\theta_{\mu \rho} \Lambda^{\rho}{ }_{\nu}-\Lambda_{\mu}{ }^{\rho} \theta_{\rho \nu}\right) p^{\nu}$ is the momentum-dependent translation. Using infinitesimal Lorentz transformation $(\Lambda=\delta+\omega)$ we get for the noncommutative space-time coordinates the following infinitesimal Lorentz transformations

$$
\widehat{x}_{\mu}^{\prime}=\widehat{x}_{\mu}+\omega_{\mu}{ }^{\nu} \widehat{x}_{\nu}+\widehat{\zeta}_{\mu},
$$

where $\widehat{\zeta}_{\mu}=\left(\omega^{\rho \nu} \theta_{\mu \rho}-\omega_{\mu}^{\rho} \theta_{\rho}^{\nu}\right) p_{\nu}$. If we perform the Weyl map $\widehat{x}_{\mu} \stackrel{W}{\rightarrow} x_{\nu}$ and observe from (47) that $\left[\widehat{x}_{\mu}, p_{\nu}\right]=i \eta_{\mu \nu} \stackrel{W}{\rightarrow}\left[x_{\mu}, p_{\nu}\right]=i \eta_{\mu \nu}$, the canonically conjugated momenta $p_{\mu}$ can be represented by the derivatives $\left(p_{\mu} \rightarrow-i \partial_{\mu}\right)$ and formula (22) is replaced by (23). Then the infinitesimal deformed Lorentz transformation, as follows from (49), imply the following change of the field operator (23)

$$
i \omega^{\mu \nu} D\left(M_{\mu \nu}\right) \varphi=\left[\omega^{\alpha \beta}\left(\delta_{\alpha}^{\mu} x_{\beta}-\delta_{\beta}^{\mu} x_{\alpha}\right)+\widehat{\zeta}^{\mu}\right] \partial_{\mu} \varphi,
$$

and we obtain that

$$
D\left(M_{\mu \nu}\right)=-i\left(x_{\mu} \partial_{\nu}-x_{\nu} \partial_{\mu}\right)-\left[\theta_{\nu}{ }^{\rho} \partial_{\rho} \partial_{\mu}+\theta_{\mu}^{\rho} \partial_{\nu} \partial_{\rho}\right] .
$$

We recall here that the formula (51) is known [15, (46]. Expressing the relation (51) for deformed differential realization as the relation between classical Poincare algebra generators we get the deformed Lorentz generators as given by nondeformed Poincare generators as follows (we recall that $P_{\mu}=P_{\mu}^{(0)}$ )

$$
M_{\mu \nu}=M_{\mu \nu}^{(0)}+\theta_{\nu}{ }^{\rho} P_{\rho} P_{\mu}+\theta_{\mu}^{\rho} P_{\nu} P_{\rho} .
$$

The covariance relations (42) describe the equality of the action on the field (22) of the Poincare algebra symmetry generators $g$ in classical (differential) space-time and quantum-mechanical (adjoint) field oscillators realizations. For arbitrary twist deformation the quantum adjoint action (24) and differential field realizations are determined by the coalgebra relations of quantum Poincare symmetry (see (4)). Several explicit examples of differential realizations $D(g)$ satisfying (42), (44) has been presented in literature, but the formulae providing $D(g)$ for arbitrary twist were not givent 4 .

\section{$5 \quad$ Braided multiplication of fields and covariant field commutator}

\subsection{The covariance of binary product of noncommutative free quantum fields}

The bilocal $\star_{\mathcal{M}}$-multiplication for canonically deformed Poincare symmetry is given by the formula (17). In the algebra of oscillators $\mathcal{H}(A(p), \cdot)$ we use the standard multiplication rule.

\footnotetext{
${ }^{4}$ See however [47] where the generalization of formula (47) for arbitrary twist is proposed
} 
We shall introduce the braid factor $\Psi_{\mathcal{M}, \mathcal{H}}$ in the physical basis of $\mathcal{M}$ and $\mathcal{H}$ in accordance with (33)

$$
\Psi_{\mathcal{M}, \mathcal{H}}\left(A(p) \underline{\otimes} e^{i q \widehat{y}}\right)=\mathcal{R}_{(2)} \triangleright e^{i q \widehat{y}} \underline{\otimes} \mathcal{R}_{(1)} \triangleright A(p) .
$$

For twist-deformed theory the multiplication prescription (32) is determined if we know the twist factor $\mathcal{F}$ and the braid $\Psi_{\mathcal{M}, \mathcal{H}}$ given by (53). The explicit form of the product (32) of fields on canonical noncommutative space-time has therefore a form

$$
\begin{aligned}
& \phi(\widehat{x}) \bullet \phi(\widehat{y})=m_{\mathcal{M} \otimes \mathcal{H}}[\phi(x) \otimes \phi(y)] \\
& =\frac{1}{(2 \pi)^{8}} \int d^{4} p \int d^{4} q \delta\left(p^{2}-m^{2}\right) \delta\left(p^{2}-m^{2}\right) \\
& e^{i p \widehat{x}}\left(\mathcal{R}_{(2)} \triangleright e^{i q \widehat{y}}\right) \underline{\otimes}\left(\mathcal{R}_{(1)} \triangleright A(p)\right) A(q), \\
& \stackrel{W}{\simeq} \frac{1}{(2 \pi)^{8}} \int d^{4} p \int d^{4} q \delta\left(p^{2}-m^{2}\right) \delta\left(p^{2}-m^{2}\right) \\
& \left(\bar{f}_{(1)} \triangleright e^{i p x}\right)\left(\bar{f}_{(2)} \mathcal{R}_{(2)} \triangleright e^{i q y}\right)\left(\mathcal{R}_{(1)} \triangleright A(p)\right) A(q),
\end{aligned}
$$

where we recall that $\mathcal{F}^{-1}=\bar{f}_{(1)} \otimes \bar{f}_{(2)}$ and by the notation $\stackrel{W}{\simeq}$ we denote the Weyl homomorphism in $\mathcal{M}$ with the $\star_{\mathcal{M}}$-product of classical Fourier exponentials representing the product $e^{i p \widehat{x}} e^{i q \widehat{y}}$. In the actions on the classical plane waves which are present in last formula (54) one uses the differential realization $D\left(g_{A}\right)$ (for deformation by canonical twist see (51)), and on the field oscillators the Poincare generators act by the quantum adjoint action (see (24), (45-46)). Before explicit calculation of braided commutator (2) we shall show that it is twist-covariant. Using formula (6) for the algebra $\Phi\left(\phi, m_{\mathcal{M} \underline{\otimes} \mathcal{H}}\right)$ of deformed free quantum fields we choost 5

$$
\begin{aligned}
g \triangleright(\phi(\widehat{x}) \bullet \phi(\widehat{y})) & =m_{\mathcal{M} \underline{\mathcal{H}}}[\Delta(g) \triangleright \phi(\widehat{x}) \otimes \phi(\widehat{y})] \\
& =\left(g_{(1)} \triangleright \phi(\widehat{x})\right) \bullet\left(g_{(2)} \triangleright \phi(\widehat{y})\right),
\end{aligned}
$$

where the quantum action (24) on the algebra $\mathcal{H}$ is used. Further $\left(\mathcal{R}_{21}=\mathcal{R}_{(2)} \otimes \mathcal{R}_{(1)}\right)$

$$
\begin{aligned}
g \triangleright\left(\mathcal{R}_{21} \triangleright[\phi(\widehat{y}) \bullet \phi(\widehat{x})]\right) & =g \triangleright\left[\left(\mathcal{R}_{(2)} \triangleright \phi(\widehat{y})\right) \bullet\left(\mathcal{R}_{(1)} \triangleright \phi(\widehat{x})\right)\right] \\
& =m_{\mathcal{M} \underline{\otimes} \mathcal{H}}\left(\Delta(g) \mathcal{R}_{21} \triangleright \phi(\widehat{y}) \otimes \phi(\widehat{x})\right) .
\end{aligned}
$$

The twisted covariance of braided commutator (2) means that

$$
g \triangleright[\phi(\widehat{x}), \phi(\widehat{y})]_{\bullet}^{B R} \equiv\left(g_{(1)} \triangleright \phi(\widehat{x})\right) \bullet\left(g_{(2)} \triangleright \phi(\widehat{y})\right)-\mathcal{R}_{21} \triangleright\left[\left(g_{(2)} \triangleright \phi(\widehat{y})\right) \bullet\left(g_{(1)} \triangleright \phi(\widehat{x})\right)\right],
$$

what requires the choice (53) of the braid factor defining $\bullet$-multiplication and the validity of the relation (see also (18))

$$
\Delta(g) \mathcal{R}_{21}-\mathcal{R}_{21} \Delta_{21}(g)=0 .
$$

\footnotetext{
${ }^{5}$ Using the covariance condition given by (41) one can choose alternatively in (55)-(57) the actions of symmetry generators on $\mathcal{H}$ by the actions on $\mathcal{M}$.
} 
Indeed, if we use the formulas (4) and (19) one shows easily that in twist-deformed theory the relation (58) is valid 6

$$
\Delta(g) \mathcal{R}_{21}-\mathcal{R}_{21} \Delta_{21}(g)=\mathcal{F}\left[\Delta_{0}(g), \tau\right] \mathcal{F}^{-1}=0
$$

\subsection{Calculation of covariant braided field commutator: braided field oscillators algebra and braided locality.}

By using (54) and (2) we shall calculate explicitly the braided commutator. We get

$$
\begin{aligned}
& {[\phi(\widehat{x}), \phi(\widehat{y})]^{B R} }=\phi(\widehat{x}) \bullet \phi(\widehat{y})-\mathcal{R}_{21} \\
& \stackrel{W}{\simeq} \frac{1}{(2 \pi)^{8}} \int d^{4} p \int d^{4} q \delta\left(p^{2}-m^{2}\right) \delta\left(q^{2}-m^{2}\right) \\
& {\left[\left(\bar{f}_{(1)} \triangleright e^{i p x}\right)\left(\bar{f}_{(2)} \mathcal{R}_{(2)} \triangleright e^{i q y}\right)\left(\mathcal{R}_{(1)} \triangleright A(p)\right) A(q)\right.} \\
&-\left(\bar{f}_{(1)} \triangleright e^{i q y}\right)\left(\bar{f}_{(2)} \mathcal{R}_{(2)} \triangleright e^{i p x}\right)\left(\mathcal{R}_{(1)} \mathcal{R}_{(2)} \triangleright A(q)\right)\left(\mathcal{R}_{(1)} \triangleright A(p)\right) .
\end{aligned}
$$

Let us consider further the canonical deformation described by the twist (11). As follows from (19) $\mathcal{R}_{21}$ depends only on the fourmomentum generators and their actions follow from the formulae

$$
P_{\mu} \triangleright e^{i p x}=p_{\mu} e^{i p x}, \quad P_{\mu} \triangleright A(p)=-p_{\mu} A(p) .
$$

In canonically deformed case the universal $\mathcal{R}$-matrix (19) acts as follows

$$
\begin{aligned}
\mathcal{R}_{21} \triangleright\left[e^{i p x} \otimes e^{i q y}\right] & =e^{i \theta^{\mu \nu} p_{\mu} q_{\nu}} e^{i p x} \otimes e^{i q y}, \\
\mathcal{R}_{21} \triangleright[A(p) \otimes A(q)] & =e^{i \theta^{\mu \nu} p_{\mu} q_{\nu}} A(p) \otimes A(q),
\end{aligned}
$$

and the braid $\Psi_{\mathcal{M}, \mathcal{H}}$ has the explicit form

$$
\Psi_{\mathcal{M}, \mathcal{H}}\left[e^{i p x} \underline{\otimes} A(q)\right]=\mathcal{R}_{(2)} \triangleright e^{i p x} \underline{\otimes} \mathcal{R}_{(1)} \triangleright A(q)=e^{-i \theta^{\mu \nu} p_{\mu} q_{\nu}} e^{i p x} \underline{\otimes} A(q) .
$$

In order to obtain $c$-number braided field commutator one should be able to factor out in (60) the binary relations satisfied by the field oscillators (the field oscillators algebra). If we use the formula (62) the required factorization in formula (60) is achieved (we use in last term of rhs of (64) the short-hand notation described in footnote 2)

$$
\begin{array}{r}
{[\phi(x), \phi(y)]_{\bullet}^{B R} \stackrel{W}{\simeq} \frac{1}{(2 \pi)^{8}} \int d^{4} p \int d^{4} q \delta\left(p^{2}-m^{2}\right) \delta\left(q^{2}-m^{2}\right) e^{i p x} e^{i q y}} \\
{\left[A(p) \star_{\mathcal{H}} A(q)-\mathcal{R}_{21} \triangleright\left(A(q) \star_{\mathcal{H}} A(p)\right)\right],}
\end{array}
$$

\footnotetext{
${ }^{6}$ In fact the relation (58) remains true and the formula (2) for covariant braided commutator of deformed fields is correct for any quasitriangular Hopf algebra with the universal $\mathcal{R}$-matrix.
} 
provided that we introduce the following multiplication for the description of binary products

$$
A(p) \star_{\mathcal{H}} A(q)=m \circ \mathcal{F} \boldsymbol{\nabla}[A(p) \otimes A(q)]=e^{\frac{i}{2} \theta^{\mu \nu} p_{\mu} q_{\nu}} A(p) A(q) .
$$

We add that the nonstandard multiplication $\star_{\mathcal{H}}$ is different from $\star_{\mathcal{M}}\left(\mathcal{F}^{-1}\right.$ in (15) is replaced by $\mathcal{F}$ ) but it is known from the literature and was used e.g. in [15].

We recall that in undeformed theory the covariant formulation of the field oscillator algebra is provided by the relation (see e.g. [44])

$$
\delta\left(p^{2}-m^{2}\right) \delta\left(q^{2}-m^{2}\right)[A(p), A(q)]=\epsilon\left(p_{0}\right) \delta\left(p^{2}-m^{2}\right) \delta^{(4)}(p+q) .
$$

The following modification of relation (66) describes the binary relation for deformed field oscillators which due to the presence of braid factor $\mathcal{R}_{21}$ is covariant under quantum symmetries and leads to $c$-number value of the braided commutator (64)

$$
\delta\left(p^{2}-m^{2}\right) \delta\left(q^{2}-m^{2}\right)\left[A(p) \star_{\mathcal{H}} A(q)-\mathcal{R}_{21} \triangleright\left(A(q) \star_{\mathcal{H}} A(p)\right)\right]=\epsilon\left(p_{0}\right) \delta\left(p^{2}-m^{2}\right) \delta^{(4)}(p+q) .
$$

If we substitute (67) into (64) we obtain that

$$
[\phi(x), \phi(y)]_{\bullet}^{B R} \stackrel{W}{\simeq} \Delta\left(x-y ; m^{2}\right)
$$

with the braided commutator for canonically deformed free quantum fields given by the known classical Pauli-Jordan function

$$
\Delta\left(x-y ; m^{2}\right)=\frac{-i}{(2 \pi)^{3}} \int \frac{d^{3} p}{\omega(\vec{p})} \sin \left[\omega(\vec{p})\left(x_{0}-y_{0}\right)\right] e^{i \vec{p}(\vec{x}-\vec{y})} .
$$

It should be noted that the choice of $\star_{\mathcal{M}}$ (see (15) for canonical case) and of the covariant braid $\Psi_{\mathcal{M}, \mathcal{H}}$ (see (133) and $(\underline{53})$ ) is necessary for getting the twist-covariant algebra of deformed field operators. The braid factor $\mathcal{R}_{21}$ which is an intertwiner in quantum quasitriangular Poincare-Hopf algebra appears in our framework on three levels:

1. in the Weyl realization of the algebra $\mathcal{M}$ as expressing the "braided commutativity" of the $\star_{\mathcal{M}}$-multiplication (see e.g. [42]; $f, h \in \mathcal{M}$ )

$$
[f, h]_{\star_{\mathcal{M}}}^{B R}:=f \star_{\mathcal{M}} h-\left(\mathcal{R}_{(2)} \triangleright h\right) \star_{\mathcal{M}}\left(\mathcal{R}_{(1)} \triangleright f\right)=0 .
$$

In particular putting $f=x_{\mu}, g=x_{\nu}$ in (70) we reproduce the space-time noncommutativity corresponding to given $\mathcal{R}$. We add that the relation (170) is covariant for any choice of $f$ and $h$ under the action of deformed Poincare generators $g=\left(P_{\mu}, M_{\mu \nu}\right)$

$$
g \triangleright[f, h]_{\star_{\mathcal{M}}}^{B R}=0 .
$$

2. in the algebra (67) of quantized field oscillators which is covariant under the action - of the symmetry generators $g$. 
3. in the algebra of deformed free quantum field $\phi$ (see (2)) and the multiplication $m_{\mathcal{M} \otimes \mathcal{H}}$, what leads to the covariance of braided field commutator (68).

We point out that the nonstandard multiplication (65) is selected by the validity of braided •-locality described by formula (68). If we look at the formula (67) from the point of view of its deformed Poincare covariance one can show that olny the braid factor $\mathcal{R}_{21}$ is required, but the choice of multiplication in $\mathcal{H}$ is not determined.

\section{Final remarks}

The aim of this paper was to study the quantum Poincare covariance in the framework of braided formulation of the theory of noncommutative quantum free fields. We restricted our considerations to binary products of such fields, but for twist-deformed noncommutative fields the extension of our formalism to n-ary associative products is straightforward. It can be shown (see e.g. [50]) that the associativity of braided products will follow from the hexagon relation satisfied by braid $\mathcal{R}_{21}$.

We stress that we employ triple twisted covariance requirement, obtained the braided form (2) of deformed field commutator, the braided form (67) of deformed oscillator algebra and braided product (32) in the algebra of deformed quantum free fields. We show that the derivation of covariant braided $c$-number field commutator describing braided locality requires the validity of the relation (67) for deformed oscillators. It should be stressed that separate elements of our construction were present in previous papers (e.g. the Weyl map (21) is used in all papers with the realization of noncommutative fields in standard Minkowski space; see also [11],[28]) but all elements of our construction occur together only in this paper.

Our explicit calculations have been given for the simplest case of canonical twist deformation, with the additional numerical phase space factors in momentum space characterizing the canonical deformation. If however the twist factor depends as well on the Lorentz generators $M_{\mu \nu}$ (see e.g. [45]), the formulae describing deformed fields are more complicated. In such a case due to the realization (40) of Lorentz generators after the Weyl map (21) the bidifferential operator describing star product $\star_{\mathcal{M}}$ depends as well on the spacetime coordinate $x_{\mu}$. Analogously the adjoint action of the braid factor $\mathcal{R}_{21}$ on oscillators in relations (67) is described effectively by the bidifferential operators in four-momentum space acting on the product of two field oscillators which are the operator-valued functions depending on the four-momenta $p_{\mu}, q_{\mu}$ ?

Important question which should be considered in the future is the application of braided-deformed free quantum fields for the description of deformed interacting QFT. For that purpose one should use the deformed version of the formulae expressing interacting

\footnotetext{
${ }^{7}$ See e.g. the derivation of nonlocal product of $\kappa$-deformed field oscillators given in [27]
} 
quantum fields in terms of free fields, within the perturbative framework. There are two ways of approaching this problem:

1. One can deform the perturbative rules for Feynman diagrams, with braid-deformed free Feynman propagators and suitably modified vertices. Such perturbative description in case of canonical deformation however without the use of braiding and quantum group symmetries was proposed firstly by Filk [29], and leads to nonlocal generalization of Dyson S-matrix formula (the nonlocality is obtained if we replace after the Weyl map the standard point-wise multiplication of fields by nonlocal $\star-$ multiplication). At present it is a challenge to derive braid-deformed counterpart of Dyson formula describing deformed perturbative S-matrix expansion (for clue in this direction see e.g. [51, [52]).

2. Other way of defining deformed interacting QFT is to modify the formulae expressing the interacting fields in terms of free asymptotic fields (they define so-called Haag expansion [53]). The basic dynamical tool for such approach is provided by perturbative solution of deformed Yang-Feldmann equation.

Finally let us observe that, the presented formalism with deformed braided fields in principle can be applied to general quasitriangular quantum deformation of free quantum fields. Such general deformations can be described by the use of twist technique only in the Drinfeld category of quasi-Hopf algebras, with nontrivial coassociator introducing nonassociative product of three field operators ([48]-[50], [54]).

$$
\phi(\widehat{x}) \bullet(\phi(\widehat{y}) \bullet \phi(\widehat{z})) \equiv \Psi_{1(23)}(\phi(\widehat{x}) \bullet \phi(\widehat{y})) \bullet \phi(\widehat{z}) .
$$

In particular because the $\kappa$-deformation of Poincare symmetries is not described by triangular Poincare-Hopf algebra, the description of $\kappa$-deformed free quantum fields covariant under $\kappa$-deformed Poincare symmetries can be described by twist only with nontrivial coassociator and requires the application of non-coassociative framework of quasi-Hopf algebras ([54], [55]). We should mention that effective application of the approach presented here to the $\kappa$-deformed field-theoretic framework is under consideration.

\section{Acknowledgements:}

We would like to thank Paolo Aschieri and Gaetano Fiore for valuable comments. The paper has been supported by Ministry of Science and Higher Education by grant NN 202331139, and at final stage by NCN grant 2011/01/B/ST2/03354.

\section{References}

[1] A. Ashtekar, J. Lewandowski, Class. Quant. Grav. 21 (2004) R53; gr-qc/0404018.

[2] Alejandro Perez, Class.Quant.Grav. 20 (2003) R43; gr-qc/0301113. 
[3] K. Becker, M, Becker, J. H. Schwarz, String theory and M-theory, Cambridge University Press, 2007.

[4] L. Randall and R. Sundrum, Phys. Rev. Lett. 83, 3370 (1999); hep-th/9905221; ibid. 83, 4690 (1999); hep-th/9906064.

[5] S. Doplicher, K. Fregenhagen, J.E. Roberts, Phys. Lett. B331 (1994) 39; Comm. Math. Phys. 172, 187 (1995); hep-th/0303037.

[6] N. Seiberg, E. Witten, JHEP 9909 (1999) 032; hep-th/9908142.

[7] P. Kosiński, J. Lukierski and M. Maślanka, Phys. Rev. D62, 025004 (2000); hep-th/9902037; Czech. J. Phys. 50, 1283 (2000); hep-th/0009120.

[8] R. Oeckl, Nucl. Phys. B581, 559 (2000); hep-th/0003018.

[9] G. Amelino-Camelia, M. Arzano, Phys. Rev. D65, 084044 (2002); hep-th/0105120.

[10] R. J. Szabo, Phys. Rep. 378, 207 (2003); hep-th/0109162.

[11] M. Dimitrijevic, L. Jonke, L. Moller, E. Tsouchnika, J. Wess and M. Wohlgenannt, Eur. Phys. J. C31, 129 (2003); hep-th/0307149.

[12] M. Chaichian, P.P. Kulish, K. Nishijma, A. Tureanu, Phys. Lett. B604 (2004) 98; hep-th/0408069.

[13] J. Wess, Lecture given at "Mathematical, Theoretical and Phenomenological Chalenges Beyond Standard Model" (29.08-2.09 2003, Vrnjacka Banja, Serbia); hep-th/0408080.

[14] P. Aschieri, Ch. Blohmann, M. Dimitrijevic, F. Meyer, P. Schupp, J. Wess, Class. Quant. Grav. 22, 3511 (2002); hep-th/0504183.

[15] G. Fiore, J. Wess, Phys. Rev. D75, 105022 (2007); hep-th/0701078.

[16] A.P. Balachandran, A. Pinzul, B. A. Qureshi, Phys. Rev. D77, 025021 (2008); arXiv 0708.1779[hep-th].

[17] S. Zakrzewski, Journ. of Phys. A27, 2079 (1994).

[18] S. Majid, H. Ruegg, Phys. Lett. Phys. Lett. B334, 348 (1994); hep-th/9405107.

[19] J. Lukierski, H. Ruegg, W.J. Zakrzewski, Annals of Phys. 243, 90 (1995); hep-th/9312153.

[20] A.P. Balachandran, G. Mangano, A. Pinzul, S. Vaidya, J.Mod.Phys.A21:3111-3126 (2006); hep-th/0508002 
[21] J.-G. Bu, H.-C. Kim, Y. Lee, C.H. Vac, J.H. Yee, Phys.Rev. D73:125001 (2006); hep-th/0603251

[22] Y. Abe, Int. J. Mod. Phys. A22, 1181 (2007); hep-th/0606183.

[23] P.P. Kulish, Proc. of Karlstad Conf. (July 2004), Satelite Conf. of IV-th European Congress of Math., ed. AMS, Contemp. Math. 391, 213 (2005); hep-th/0606056.

[24] M. Daszkiewicz, J. Lukierski, M. Woronowicz, Mod. Phys. Lett. A23, 9 (2008); hep-th/0703200.

[25] M. Daszkiewicz, J. Lukierski, M. Woronowicz, Phys. Rev. D77, 105007 (2008); arXiv: 0708.1561 [hep-th].

[26] C. A. S. Young, R. Zegers, Nucl. Phys. B797 (2008) 537; arXiv:0711.2206 [hep-th].

[27] T. R. Govindarajan, K. S. Gupta, E. Harikumar, S. Meljanac, D. Meljanac, Phys. Rev. D80, 025014 (2010); arXiv:0903.2355 [hep-th].

[28] G. Fiore, Proc. of Conf. "Noncommutative Structures In Mathematics and physics", Brussels 22-26/7/2008, Ed. S. Caenepeel, at all., Royal Flemish Academy of Belgium for Sciences and Arts, Brussels, 2010, pp.163-177; arXiv:1007.0885; G. Fiore, in "Quantum Field Theory and Beyond", Proceedings of the Symposium in honor ofWolfhart Zimmermann's 80th birthday, (Ringberg Castle 02/2008). Ed. E. Seiler, K. Sibold, World Scient. Publi. Co. 2008, 64-84; arXiv:0809.4507; and G. Fiore, Jour. Phys. A43: 155401 (2010); arXiv:0811.0773 [hep-th].

[29] T. Filk, Phys. Lett. B376, 53 (1996)

[30] H. Grosse, G. Lechner, JHEP 0809:131 (2008); arXiv:0808.3459 [hep-th]; JHEP 0711.012 (2007) arXiv:0706.3992 [hep-th].

[31] G. Piacitelli, Commun. Math. Phys.295, 701 (2010); arXiv:0902.0575 [hep-th].

[32] A. B. Zamolodchikov, A.B Zamolodchikov, Ann. Phys. 120, 253 (1979).

[33] L. Faddeev, Sov. Sci. Rev. C1, 107 (1980).

[34] W. Pusz, S. L. Woronowicz, Rep. Math. Phys. 27, 231 (1989).

[35] M. Chaichian, P. Kulish, J. Lukierski, Phys. Lett. B262, 43 (1991).

[36] G. Fiore, J. Math. Phys. 39, 3437 (1998); q-alg/9610005.

[37] S. Majid, Mod. Phys. A5, 1 (1990).

[38] E. Joung, J. Mourad, JHEP 0705:098 (2007); hep-th/0703245 
[39] A. P. Balachandran, A. Ibort, G. Marmo and M. Martone, JHEP 1103.057 (2011); arXiv:1009.5136 [hep-th].

[40] G. Piacitelli, Proc. of Corfu Summer Institute, Sept. 2010, Proc. of Science CNCFG 2010 (in electronic form); arXiv:1103.3405 [hep-th].

[41] A. Tureanu, Phys. Lett. B638, 296 (2006); hep-th/0603219.

[42] P. Aschieri, F. Lizzi, P. Vitale, Phys. Rev. D77:025037 (2008); arXiv:0708.3002[hepth].

[43] V. G. Drinfeld, Sov. Math. Dokl. 28, 667 (1983).

[44] N.N. Bogolubov, D.W. Shirkov, Introduction into the theory of quantized fields, first Edition in Russian in 1957 (Nauka, Moscow-Leningrad); English version AddisonWesley, 1982.

[45] J. Lukierski, M. Woronowicz, Phys. Lett. B633: 116-125 (2006); hep-th/0508083.

[46] F. Koch, E. Tsouchnika, Nucl. Phys. B717, 387 (2005); hep-th/0409012.

[47] A. Borowiec, A. Pachol, SIGMA 6:086 (2010); arXiv:1005.4429 [hep-th].

[48] V.G. Drinfeld, Leningrad Math. J. 1, 1419 (1990).

[49] E. J. Beggs, S. Majid; arXiv:math/0506453.

[50] S. Majid, Foundations of quantum group theory, Cambridge Univ. Press (1996), Sect. 9.

[51] Y. Sasai, N. Sasakura, Prog. Theor.Phys. 118, 785 (2007): arXiv:0704.0822 [hep-th].

[52] R. Oeckl, Comm. Math. Phys. 217, 451 (2001): hep-th/9906225.

[53] R. Haag, On quantum field theories, Dan. Math. Fys. Medd. 29, No12 (1955)

[54] C.A.S. Young, R. Zegers, Comm. Math. Phys. 298, 585 (2010), arXiv:0812.3257[hepth].

[55] C.A.S. Young, R. Zegers, Nucl. Phys. B809, 439 (2009); arXiv:0807.2745[hep-th]. 